Pacific

Journal of

Mathematics

ON REPRESENTATIONS OF AFFINE TEMPERLEY-LIEB ALGEBRAS, II

K. Erdmann and R.M. Green

Volume $191 \quad$ No. 2

December 1999 


\title{
ON REPRESENTATIONS OF AFFINE TEMPERLEY-LIEB ALGEBRAS, II
}

\author{
K. Erdmann and R.M. Green
}

\begin{abstract}
We study some non-semisimple representations of affine Temperley-Lieb algebras and related cellular algebras. In particular, we classify extensions between simple standard modules. Moreover, we construct a completion which is an infinite dimensional cellular algebra.
\end{abstract}

\section{Introduction.}

The affine Temperley-Lieb algebra, $T L\left(\widehat{A}_{n-1}\right)$, is an infinite dimensional algebra which occurs as a quotient of the Hecke algebra associated to a Coxeter system of type $\widehat{A}_{n-1}$. It occurs naturally in the context of statistical mechanics [10], [11], and may be thought of as an algebra of diagrams (see $[\mathbf{3}, \S 4])$. In $[\mathbf{6}]$, the second author used the diagram calculus and the theory of cellular algebras as described by Graham and Lehrer [4] to classify and characterise most finite dimensional irreducible modules for $T L\left(\widehat{A}_{n-1}\right)$ and for a larger algebra of diagrams, $D_{n}$. In fact, all simple modules for these algebras are finite dimensional; this follows for example from [12, 13.10.3].

In general, a cellular algebra produces a natural class of modules, called standard modules or cell modules. Special cases of these include Weyl modules and Specht modules. They are not semisimple in general, but one obtains all irreducible modules as simple quotients of appropriate standard modules. The algebras $D_{n}$ (and $T L\left(\widehat{A}_{n-1}\right)$ ) have many finite dimensional cellular quotients. These were used as a main tool in [6], where they were called $q$-Jones algebras.

In this paper, we extend the results of [6] by studying non-semisimple finite dimensional modules for $D_{n}$, and also the remaining simple modules which were not considered in [6]. Moreover, we construct completions for the algebras $D_{n}$ which are infinite dimensional cellular algebras. Our techniques also work for the algebra $T L\left(\widehat{A}_{n-1}\right)$, but we will not always make this explicit. We assume $K$ is an algebraically closed field and $v \in K^{*}$ is such that $\delta=v+v^{-1}$ is non-zero. Unless otherwise stated, we are only concerned with finite dimensional modules.

After reviewing some of the results from [6] in $\S 2$, we classify extensions between simple standard modules for $D_{n}$ in $\S 3$. As a consequence we can 
describe all finite dimensional modules of $D_{n}$ which lie in blocks $B_{q}$ where $q$ is such that the corresponding $q$-Jones algebra is semisimple; such blocks exist in abundance. In this case every finite dimensional module in such a block is a direct sum of uniserial modules, and an indecomposable summand has only one type of composition factor.

In $\S 4$, we construct an infinite dimensional cellular algebra, $D_{n}^{+}$, which is spanned by a set of "positive" diagrams. The smooth modules for various procellular completions of this algebra (in the sense of [7]) provide all the uniserial modules described in $\S 3$, and the algebra $D_{n} / I_{0}$ embeds densely in any of these procellular completions. This means that the algebra $D_{n} / I_{0}$ is in some sense almost cellular.

In $\S 5$ we study extensions of standard modules for cellular algebras more generally. Here we follow the approach of S. König and C. Xi [9] where a more algebraic treatment of these algebras is given. In $\S 6$, we use these results to determine extensions between simple standard modules for the associated $q$-Jones algebras, over arbitrary characteristic, with $\delta \neq 0$ but otherwise arbitrary. Finally in $\S 7$, we classify the remaining simple modules for $D_{n}$.

Note that our approach is to make use of the $q$-Jones algebras, which are deformations of certain quotients of Jones' annular algebras as introduced in [8]. All these algebras are finite dimensional and cellular, with an explicit cell basis which can be dealt with by elementary algebra [6, §3].

After submitting this paper for publication, the authors received a copy of Graham and Lehrer's paper [5] which derives the results of [6] independently and using different methods. The focus of [5] is rather different from this paper: The main results of [5] give a complete determination of the multiplicities of the composition factors of all the cell modules, subject to some restrictions on the ground ring. In this paper, we are concerned with the simple standard modules, and our central objects of study are instead the non-semisimple modules which have filtrations by simple standard modules.

\section{Algebras of diagrams related to affine Temperley-Lieb algebras.}

In $\S 2$ we recall from $[6]$ the definitions of various algebras of diagrams which are related to $T L\left(\widehat{A}_{n-1}\right)$.

\subsection{Affine $n$-diagrams.}

We assume that $K$ is an algebraically closed field which contains a nonzero element $v$. Let $\delta=[2]=v+v^{-1}$; we assume that $\delta$ is non-zero in $K$. We consider the affine Temperley-Lieb algebra as a subalgebra of an algebra $D_{n}$ as defined in [6]. This is defined in terms of diagrams as follows.

2.1.1. An affine $n$-diagram, where $n \in \mathbb{Z}$ satisfies $n \geq 3$, consists of two infinite horizontal rows of nodes lying at the points $\{\mathbb{Z} \times\{0,1\}\}$ of $\mathbb{R} \times$ 
$\mathbb{R}$, together with certain curves, called edges, which satisfy the following conditions:

(i) Every node is the endpoint of exactly one edge.

(ii) Any edge lies within the strip $\mathbb{R} \times[0,1]$.

(iii) If an edge does not link two nodes then it is an infinite horizontal line which does not meet any node. Only finitely many edges are of this type.

(iv) No two edges intersect each other.

(v) An affine $n$-diagram must be invariant under shifting to the left or to the right by $n$.

2.1.2. By an isotopy between diagrams, we mean one which fixes the nodes and for which the intermediate maps are also diagrams which are shift invariant. We will identify any two diagrams which are isotopic to each other, so that we are only interested in the equivalence classes of affine $n$-diagrams up to isotopy. This has the effect that the only information carried by edges which link two nodes is the pair of vertices given by the endpoints of the edge.

2.1.3. Because of the condition (v) in 2.1.1, one can also think of affine $n$-diagrams as diagrams on the surface of a cylinder, or within an annulus, in a natural way. Unless otherwise specified, we shall henceforth regard the diagrams as diagrams on the surface of a cylinder with $n$ nodes on top and $n$ nodes on the bottom. From now on, we will call the affine $n$-diagrams "diagrams" for short, when the context is clear. Under this construction, the top row of nodes becomes a circle of $n$ nodes on one face of the cylinder, which we will refer to as the top circle. Similarly, the bottom circle of the cylinder is the image of the bottom row of nodes.

Example 2.1.4. An example of an affine $n$-diagram for $n=4$ is given in Figure 1. The dotted lines denote the periodicity, and should be identified to regard the diagram as inscribed on a cylinder.

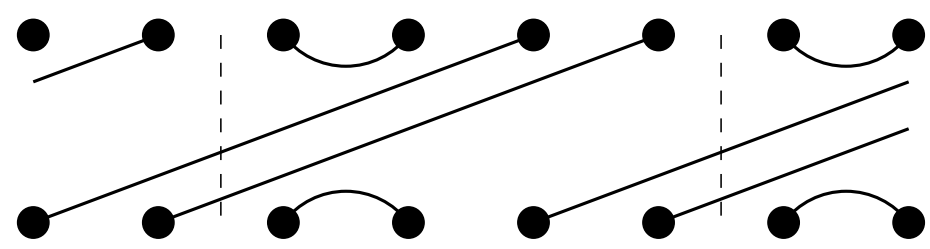

Figure 1. An affine 4-diagram.

2.1.5. An edge of the diagram $D$ is said to be vertical if it connects a point in the top circle of the cylinder to a point in the bottom circle, and horizontal if it connects two points in the same circle of the cylinder. Following [4], we will also call the vertical edges "through-strings". 
2.1.6. Two diagrams, $A$ and $B$ "multiply" in the following way, which was described in $[3, \S 4.2]$. Put the cylinder for $A$ on top of the cylinder for $B$ and identify all the points in the middle row. This produces a certain (natural) number $x$ of loops. Removal of these loops forms another diagram $C$ satisfying the conditions in 2.1.1. The product $A B$ is then defined to be $\delta^{x} C$. It is clear that this defines an associative multiplication.

2.1.7. Let $R=R^{\prime}\left[v, v^{-1}\right]$ be the ring of Laurent polynomials over an integral domain. We define the associative algebra $D_{n}$ over $R$ to be the $R$-linear span of all the affine $n$-diagrams, with multiplication given as above. Similarly, we may define $D_{n}$ over the algebraic closure of the field of fractions of $R$, or more generally, over $K$.

\subsection{Generators and relations.}

The diagram $u$ defined below plays an important rôle in classifying the modules we are interested in.

2.2.1. Denote by $\bar{i}$ the congruence class of $i$ modulo $n$, taken from the set $\mathbf{n}:=\{1,2, \ldots, n\}$. We index the nodes in the top and bottom circles of each cylinder by these congruence classes in the obvious way.

2.2.2. The diagram $u$ of $D_{n}$ is the one satisfying the property that for all $j \in \mathbf{n}$, the point $j$ in the bottom circle is connected to point $\overline{j+1}$ in the top circle by a vertical edge taking the shortest possible route.

In the case $n=4$, the element $u$ is as shown in Figure 2 .

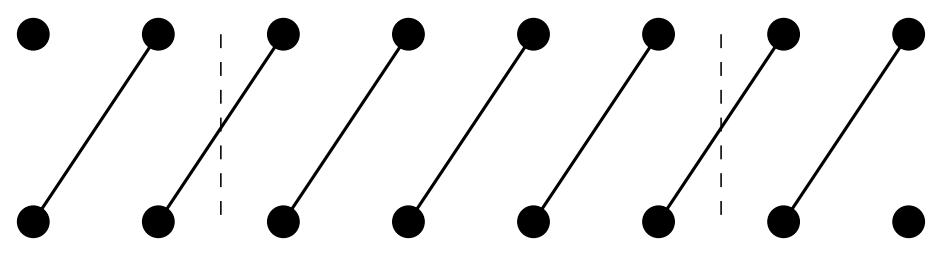

Figure 2. The element $u$ for $n=4$.

2.2.3. The diagram $E_{i}$ (where $1 \leq i \leq n$ ) has a horizontal edge of minimal length connecting $\bar{i}$ and $\overline{i+1}$ in each of the circles of the cylinder, and a vertical edge connecting $\bar{j}$ in the top circle to $\bar{j}$ in the bottom circle whenever $\bar{j} \neq \bar{i}, \overline{i+1}$.

A typical cylindrical representation of a diagram $E_{i}$ is shown in Figure 3. 


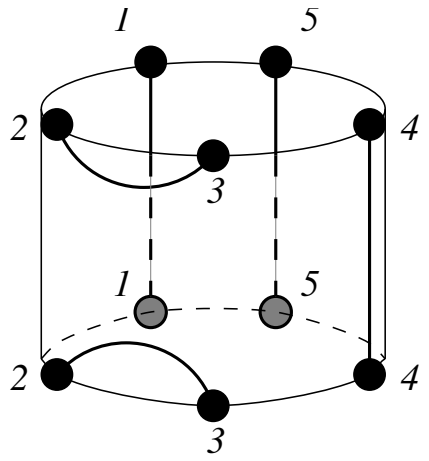

Figure 3. The element $E_{2}$ for $n=5$.

Proposition 2.2.4. The algebra $D_{n}$ is generated by elements

$$
E_{1}, \ldots, E_{n}, u, u^{-1} \text {. }
$$

It is subject to the following defining relations:

$$
\begin{aligned}
E_{i}^{2} & =\delta E_{i}, \\
E_{i} E_{j} & =E_{j} E_{i}, \quad \text { if } \bar{i} \neq \overline{j \pm 1}, \\
E_{i} E_{\overline{i \pm 1}} E_{i} & =E_{i} . \\
u E_{i} u^{-1} & =E_{\overline{i+1}}, \\
\left(u E_{1}\right)^{n-1} & =u^{n} \cdot\left(u E_{1}\right) .
\end{aligned}
$$

Proof. This is [6, Proposition 2.3.7].

The affine Temperley-Lieb algebra embeds in the algebra $D_{n}$ as a subalgebra.

Proposition 2.2.5. The algebra $T L\left(\widehat{A}_{n-1}\right)$ is the subalgebra of $D_{n}$ spanned by diagrams, $D$, with the following additional properties:

(i) If $D$ has no horizontal edges, then $D$ is the identity diagram, in which point $j$ in the top circle of the cylinder is connected to point $j$ in the bottom circle for all $j$.

(ii) If $D$ has at least one horizontal edge, then the number of intersections of $D$ with the line $x=i+1 / 2$ for any integer $i$ is an even number.

Equivalently, $T L\left(\widehat{A}_{n-1}\right)$ is the unital subalgebra of $D_{n}$ generated by the elements $E_{i}$ and subject to relations (1)-(3) of Proposition 2.2.4.

Proof. See [6, Definition 2.2.1, Proposition 2.2.3].

One would like to understand the finite dimensional simple modules for the affine Temperley-Lieb algebras $T L\left(\widehat{A}_{n-1}\right)$. These are more or less the same as the finite dimensional modules for $D_{n}$, as is explained in detail in 
$[6, \S 4]$. The above relations show that the element $u^{n}$ is central in the algebra $D_{n}$, so by Schur's Lemma, it acts as a scalar on any finite dimensional simple module, and the scalar must be invertible in $K$.

The finite dimensional simple $D_{n}$-modules over $K$ are precisely the simple modules for quotient algebras $D_{n} /\left\langle u^{n}-q\right\rangle$ where $q \in K$ is invertible. These are essentially the $q$-Jones algebras of $[\mathbf{6}]$, and are finite dimensional. In [6], it was shown that the $q$-Jones algebras are cellular, which allowed one to classify the irreducible representations; we will summarize these results below in $§ 2.5$. In this paper, we will finish this classification and study more general finite dimensional modules.

For our approach, the finite dimensional $q$-Jones algebras are the starting point. The representation theory of these algebras is in some sense similar to the representation theory of finite dimensional quotients of the polynomial ring $K[X]$, and may be treated with elementary methods.

\subsection{The oriented subalgebra $O_{n}$.}

We also introduce a subalgebra $O_{n}$ of $D_{n}$, called the "oriented subalgebra". This is related to $D_{n}$ in the same way as the alternating groups are related to the symmetric groups. The algebra $O_{n}$ is defined in terms of number of intersections with certain vertical lines; for this purpose, we do not count a curve tangent to a line as an intersection.

2.3.1. Define $O_{n}$ to be the $K$-submodule of $D_{n}$ spanned by diagrams $D$ such that the number of intersections of $D$ with the line $x=i+1 / 2$ for any integer $i$ is an even number.

Such diagrams are said to have the even intersection property. Conversely, if all the numbers of intersections of a diagram $D$ with the lines $x+1 / 2$ are odd, $D$ is said to have the odd intersection property.

Lemma 2.3.2. Any diagram $D$ of $D_{n}$ has the odd intersection property or the even intersection property.

Furthermore, if $D$ has the even (respectively, odd) intersection property, then $u . D$ has the odd (respectively, even) intersection property.

Proof. This was shown in [6, Proposition 2.3.4].

Lemma 2.3.3. The $K$-module $O_{n}$ is a subalgebra of $D_{n}$, and is generated by $T L\left(\widehat{A}_{n-1}\right)$ and the elements $\left\{u^{2 m}: m \in \mathbb{Z}\right\}$.

Proof. This is [6, Lemma 2.3.3].

\subsection{Annular involutions.}

Let $D$ be an affine $n$-diagram associated to the algebra $D_{n}$. Throughout $\S 2.4$ we are only concerned with diagrams $D$ with $t>0$ vertical edges. (We deal with the case $t=0$ in $\S 7$.) If $t>0$, we can define the winding number $w(D)$ as follows. 
2.4.1. Let $D$ be as above. Let $w_{1}(D)$ be the number of pairs $(i, j) \in$ $\mathbb{Z} \times \mathbb{Z}$ where $i>j$ and $\bar{j}$ in the bottom circle of $D$ is joined to $\bar{i}$ in the top circle of $D$ by an edge which crosses the "seam" $x=1 / 2$. We then define $w_{2}(D)$ similarly but with the condition that $i<j$, and we define $w(D)=w_{1}(D)-w_{2}(D)$.

Remark 2.4.2. At least one of $w_{1}(D)$ or $w_{2}(D)$ is 0 , and $w(D)$ is always finite, although it is unbounded for a fixed value of $n$.

The winding numbers of the diagrams in Figures 1,2 and 3 are 2, 1 and 0 , respectively. Also note that $w\left(u^{n}\right)=n$ for any $n \in \mathbb{Z}$.

Graham and Lehrer [5] introduce "asymptotic modules" $W_{0}, W_{\infty}$ instead of using winding numbers.

We recall the definition of an annular involution of the symmetric group from [4, Lemma 6.2].

2.4.3. An involution $S \in \mathcal{S}_{n}$ is annular if and only if for each pair $i, j$ interchanged by $S(i<j)$, we have

(a) $S[i, j]=[i, j]$ and

(b) $[i, j] \cap \operatorname{Fix} S=\emptyset$ or $\operatorname{Fix} S \subseteq[i, j]$.

We write $S \in I(t)$ if $S$ has $t$ fixed points, and we write $S \in \operatorname{Ann}(\mathbf{n})$ if $S$ is annular. In case $t=n$ we view the identity permutation as an annular involution.

Using the concept of winding number, we have the following bijection.

Proposition 2.4.4. Let $D$ be a diagram for $D_{n}$ with $t$ vertical edges $(t>0)$. Define $S_{1}, S_{2} \in \operatorname{Ann}(\mathbf{n}) \cap I(t)$ and $w \in \mathbb{Z}$ as follows.

The involution $S_{1}$ exchanges points $i$ and $j$ if and only if $i$ is connected to $j$ in the top circle of $D$. Similarly $S_{2}$ exchanges points $i$ and $j$ if and only if $i$ is connected to $j$ in the bottom circle of $D$. Set $w=w(D)$.

Then this procedure produces a bijection between diagrams $D$ with at least one vertical edge and triples $\left[S_{1}, S_{2}, w\right]$ as above.

Proof. See [6, Lemma 3.2.4].

2.4.5. For $S_{1}, S_{2} \in \operatorname{Ann}(\mathbf{n}) \cap I(t)$ where $t>0$, we define $r\left(S_{1}, S_{2}\right)$ to be the smallest nonnegative integer satisfying $\left[S_{1}, S_{2}, r\left(S_{1}, S_{2}\right)\right] \in O_{n}$.

Remark 2.4.6. The integer $r\left(S_{1}, S_{2}\right)$ is necessarily 0 or 1 (see [6, Definition $3.2 .5])$.

2.4.7. For $t \geq 0$ and $t \leq n$ with $t \equiv n(\bmod 2)$ let $I_{t}$ be the span of all diagrams with at most $t$ through-strings. This is an ideal of $D_{n}$. If $t>0$ it is spanned, modulo $I_{t-2}$, by all elements $\left[S_{1}, S_{2}, w\right]$ where $S_{1}, S_{2} \in$ $\operatorname{Ann}(\mathbf{n}) \cap I(t)$.

We also define $I_{-1}=0$ for convenience. 
2.4.8. Notation. Suppose $f(X) \in K[X]$ is a polynomial, and let $S_{1}, S_{2} \in$ $\operatorname{Ann}(\mathbf{n}) \cap I(t)$ for $t>0$. Then we write

$$
f\left(S_{1}, S_{2}\right)
$$

for the element of $I_{t}$ obtained from $f(X)$ by substituting $\left[S_{1}, S_{2}, k\right]$ for $X^{k}$.

\section{5. $q$-Jones algebras.}

In [6], finite dimensional quotient algebras $J_{q}(n)$ of $D_{n}$ (for $n$ odd) and $O_{n}$ (for $n$ even) were introduced, called $q$-Jones algebras. These are cellular, and by using structural results on cellular algebras, the finite dimensional irreducible modules were classified for the case when $v$ is an indeterminate. Moreover, by restriction these give all finite dimensional irreducible modules of the affine Temperley-Lieb algebra, letting $q \in K^{*}$ vary.

The ordinary Jones algebra (corresponding to $q=1$ ) can be simply described by using a homomorphism from $D_{n}$ to the Brauer algebra [8]. There may exist a suitable deformation of the Brauer algebra which plays a similar rôle for the $q$-Jones algebra, but we do not pursue this here.

Here we allow $v$ more generally to be an element in $K^{*}$ (which may or may not be an indeterminate) but we assume that $\delta$ is non-zero. In this case, the $q$-Jones algebra need not be semisimple, but it is still cellular. It therefore has standard modules, and all simple modules occur as quotients of these standard modules. Therefore we study more generally the finite dimensional modules for $D_{n}$ and their restrictions to $T L\left(\widehat{A}_{n-1}\right)$ which are standard modules for a $q$-Jones algebra, for some $q \in K^{*}$.

2.5.1. We recall the definition of the $q$-Jones algebra and of its standard modules. Since $u^{n}$ is central in $D_{n}$ it acts as scalar multiplication on finite dimensional simple modules. Therefore, let $\omega(q)$ be the ideal of $D_{n}$ generated by $u^{n}-q$, for $q \in K^{*}$. It is equal, modulo $I_{0}$, to the span of the set

$\left\{\left[S_{1}, S_{2}, w+t s\right]-q^{s}\left[S_{1}, S_{2}, w\right]: S_{1}, S_{2} \in \operatorname{Ann}(\mathbf{n}) \cap I(t), t \in \mathcal{T}(n), w, s \in \mathbb{Z}\right\}$.

Following [6], we introduce the $q$-Jones algebras as follows. If $n$ is odd define $J_{q}(n):=D_{n} / \omega(q)$. On the other hand, if $n$ is even we define

$$
J_{q}(n):=O_{n} /\left(O_{n} \cap\left(\omega(q)+I_{0}\right)\right) .
$$

These are finite dimensional cellular algebras, and the restrictions of simple modules for $J_{q}(n)$ (viewed as $D_{n^{-}}$or $O_{n}$-modules) give all finite dimensional simple modules for the affine Temperley-Lieb algebra, except simples $M$ such that $I_{0} M=M$ in case $n$ is even. (We will deal with these in $\S 7$; see also [5], [10].)

We will now describe the cellular structure and define the standard modules. Proofs may be found in [6, Proposition 3.4.2, Proposition 3.5.6]. 
2.5.2. Assume first that $n$ is odd. Let $\mathcal{T}(n)=\{1,3, \cdots, n-2, n\}$. For $t \in \mathcal{T}(n)$, let $X^{t}-q=\prod_{i=1}^{t}\left(X-r_{i}(t, q)\right)$, choosing some labelling for the roots. Let

$$
f^{(t, j)}(X)=\prod_{i>j}\left(X-r_{i}(t, q)\right), \quad(1 \leq j \leq t),
$$

and set $f^{(t, 0)}(X)=1$. Then a cell datum for $J_{q}(n)$ over $K$ is given by $(\Lambda, M, C, *)$ where:

(a) $\Lambda=\{(t, j): t \in \mathcal{T}(n), j \in \mathbf{t}\}$, the set of weights, ordered lexicographically.

(b) For $(t, j) \in \Lambda$, let $M(t, j)=\operatorname{Ann}(\mathbf{n}) \cap I(t)$.

(c) If $S_{1}, S_{2} \in M(t, j)$ define

$$
C_{S_{1}, S_{2}}^{(t, j)}:=f^{(t, j)}\left(S_{1}, S_{2}^{*}\right) .
$$

(d) $\left[S_{1}, S_{2}, w\right]^{*}=\left[S_{2}^{*}, S_{1}^{*}, w\right]$ where $S^{*}=w_{0} S w_{0}^{-1}$.

2.5.3. Assume $t>0$ and $t \equiv n(\bmod 2)$. The intersection $\omega(q) \cap O_{n}$ is spanned by the set

$$
\left\{\left[S_{1}, S_{2}, r\left(S_{1}, S_{2}\right)+2 w+t s\right]-q^{s}\left[S_{1}, S_{2}, r\left(S_{1}, S_{2}\right)+2 w\right]\right\}
$$

where $r\left(S_{1}, S_{2}\right)$ is as in 2.4.5, $S_{1}, S_{2} \in I(t)$ and $w, s \in \mathbb{Z}$.

Assume $n$ is even. Then $J_{q}(n)=O_{n} /\left(O_{n} \cap\left(\omega(q)+I_{0}\right)\right)$ is cellular. The cell data are as before but $\mathcal{T}(n)$ is replaced by $\mathcal{T}^{\prime}(n)=\{1,2, \cdots, n / 2\}$, and in the polynomial, $\left[S_{1}, S_{2}, r\left(S_{1}, S_{2}\right)+2 k\right]$ is substituted for $X^{k}$.

To see this, observe first that $J_{q}(n)$ has a basis the cosets of

$$
\left\{\left[S_{1}, S_{2}, r\left(S_{1}, S_{2}\right)+2 k\right]: 0 \leq k \leq t / 2\right\},
$$

where $S_{1}, S_{2} \in \operatorname{Ann}(\mathbf{n}) \cap I(t)$. Fix $S_{1}, S_{2}$ and define a $K$-linear map $K[X] \rightarrow$ $J_{q}(n)$ by

$$
X^{k} \rightarrow\left[S_{1}, S_{2}, r\left(S_{1}, S_{2}\right)+2 k\right] .
$$

This map takes $X^{t / 2}-q$ to $\left(u^{n}-q\right)\left[S_{1}, S_{2}, r\left(S_{1}, S_{2}\right)\right] \in \omega(q)$ and hence it induces a monomorphism $K[X] /\left(X^{t / 2}-q\right) \rightarrow J_{q}(n)$. Then the set-up in 2.5.2 with $X^{t / 2}-q$ instead of $X^{t}-q$ gives us a cell datum.

2.5.4. The filtration of $D_{n}$ by the ideals $I_{t}$ induces a filtration of $J_{q}(n)$ by ideals which we also denote by $I_{t}$. This chain of ideals has a refinement. Let $J_{(t, j)}$ be the span of the set

$$
\left\{C_{S_{1}, S_{2}}^{\lambda}: \lambda \leq(t, j), S_{1}, S_{2} \in M(\lambda)\right\} .
$$

This is an ideal and we have

$$
I_{t-2} \subset J_{(t, 1)} \subset \ldots \subset J_{(t, t-1)} \subset J_{(t, t)}=I_{t} .
$$

Each quotient, as a left module, is a direct sum of standard modules (for the general definition, see [4] or [9]). If $\lambda=(t, j)$ then $W(\lambda)$ can be taken as as the span of the cosets of $\left\{C_{S, T}^{\lambda}: S \in M(\lambda)\right\}$ where $T \in M(\lambda)$ is fixed. 
We will also call it a standard module if we view it as a module for $D_{n}$ or $T L\left(\widehat{A}_{n-1}\right)$.

2.5.5. We note that any labelling of the roots of $X^{t}-q$ (respectively, $X^{t / 2}-q$ ) gives rise to such chain of ideals, so in general there are many such chains. It is also clear from $[\mathbf{6}, \S 4]$ that standard modules $W(t, j)$ and $W(t, k)$ are isomorphic if and only if the associated roots $r_{j}$ and $r_{k}$ are equal. Moreover, the standard modules depend only on the roots and not on the particular cell chain. We will therefore sometimes write $W(t, \alpha)$ if $\alpha$ is a root of $X^{t}-q$ (respectively, $X^{t / 2}-q$ ).

2.5.6. We recall the parametrization of the simple modules for a cellular algebra. For each $\lambda$ there is an associated bilinear form $\phi_{\lambda}$. Let $\Lambda_{0}:=\{\lambda$ : $\left.\phi_{\lambda} \neq 0\right\}$. If $\lambda \in \Lambda_{0}$ then $W(\lambda)$ has a simple top, which we denote by $L(\lambda)$. The set $\left\{L(\lambda): \lambda \in \Lambda_{0}\right\}$ is a full set of simple modules for a cellular algebra $[4]$.

For $q$-Jones algebras in general, every standard module is isomorphic to some $W(\lambda)$ with $\lambda \in \Lambda_{0}$; this is not hard to see, and this will follow from our results in $\S 6$. In particular all standard modules have a simple top, and the distinct simple modules are in 1-1 correspondence with the distinct roots of the polynomials $X^{t}-q$ for $t \in \mathcal{T}(n)$.

2.5.7. For $n$ even, there is also a cellular algebra $D_{n}(q):=D_{n} /(\omega(q)+$ $I_{0}$ ), with cell structure as in 2.5.2 above where $\mathcal{T}(n)=\{2,4, \cdots, n\}$. This contains the algebra defined in 2.5.3 as a subalgebra of half the dimension. It is also convenient to define $D_{n}(q):=J_{q}(n)$ in the case where $n$ is odd.

2.5.8. Similarly if $n$ is odd then there is a cellular finite dimensional algebra $O_{n}(q):=O_{n} /\left(\omega(q) \cap O_{n}\right)$. By considering the basis given in 2.5.2 we see that this is also cellular, with cell datum as in 2.5.2 except that the starting polynomial is $X^{t}-q^{2}$, and one substitutes $\left[S_{1}, S_{2}, r\left(S_{1}, S_{2}\right)+2 k\right]$ into $X^{k}$. By definition, $O_{n}(q)$ is a subalgebra of $J_{q}(n)$. Both have the same dimension, so they are isomorphic, but the isomorphism is not canonical.

Remark 2.5.9. The algebra $J_{q}(n)$ has a subalgebra $\Gamma_{n}(q)$ given as

$$
\frac{\Gamma}{\omega(q) \cap \Gamma} \quad \text { or } \quad \frac{\Gamma}{\left(\omega(q)+I_{0}\right) \cap \Gamma}
$$

where $\Gamma=T L\left(\widehat{A}_{n-1}\right)$. Here the inclusion is compatible with the cell structure although one must replace $I_{n} / I_{n-2}$ by $K$.

Remark 2.5.10. Suppose $n$ is even. If $q \neq 1$ then $I_{0} \subset \omega(q)$ automatically, but if $q=1$ then $I_{0}$ must be factored out. We will deal with this in $\S 7$.

\section{Non-semisimple modules for $D_{n}$.}

A natural question to ask is: are there any non-trivial extensions between simple modules for $D_{n}$ ? 
There are two possible types. First there may be extensions which are not modules for any $D_{n}(q)$. Second, there may be extensions which already occur for $D_{n}(q)$-modules. We will call these extensions of the first (respectively, second) type. We start with the first type. All the modules in $\S 3$ are $D_{n}$-modules unless otherwise stated. We write $\operatorname{Hom}(-,-)$ instead of $\operatorname{Hom}_{A}(-,-)$ and similarly for $\operatorname{Ext}^{1}$ if the algebra $A$ is clear from the context.

\subsection{Some properties of extensions.}

3.1.1. We consider finite dimensional $D_{n}$-modules. Since $u^{n}$ is central in $D_{n}$, such modules have a natural block decomposition. For $q \in K^{*}$, define $B_{q}$ to be the category of finite dimensional $D_{n}$-modules on which some power of $u^{n}-q$ acts as zero, with maps all $D_{n}$-homomorphisms. That is, a finite dimensional $D_{n}$-module belongs to $B_{q}$ if and only if $q$ is the only eigenvalue of $u^{n}$ on $M$.

Thus every finite dimensional $D_{n}$-module $M$ is a direct sum

$$
M=M_{1} \oplus M_{2} \oplus \cdots \oplus M_{r} .
$$

Here, $M_{i}$ belongs to the block $B_{q_{i}}$ where $q_{1}, \ldots, q_{r}$ are the distinct eigenvalues of $u^{n}$ on $M$. (Namely, take $M_{i}$ to be the kernel of $\left(u^{n}-q_{i}\right)^{a_{i}}$, where $u^{n}$ has minimal polynomial $\prod_{i=1}^{r}\left(X-q_{i}\right)^{a_{i}}$; this is a $D_{n}$-module.)

Suppose $M, M^{\prime}$ are finite dimensional modules for $D_{n}$ which belong to blocks $B_{a}$ and $B_{b}$ respectively.

Lemma 3.1.2. Suppose there is a short exact sequence

$$
0 \rightarrow M^{\prime} \rightarrow N \rightarrow M \rightarrow 0
$$

of modules for $D_{n}$, where $N$ is indecomposable. Then $u^{n}$ has the same eigenvalue on $M$ and on $M^{\prime}$.

Proof. If $a \neq b$ then $N$ would have two distinct non-zero summands in different blocks.

3.1.3. Lemma 3.1 .2 now allows us to restrict our attention to the case $a=b=q$.

Lemma 3.1.4. Suppose $M$ has simple top $L(\lambda)$ and assume $L(\lambda)$ does not occur as a composition factor of $M^{\prime}$. Then there is no exact sequence $0 \rightarrow$ $M^{\prime} \rightarrow N \rightarrow M \rightarrow 0$ such that $u^{n}-q$ does not act trivially on $N$.

Proof. Suppose this is false. Let $\psi$ denote multiplication by $u^{n}-q$. This is a $D_{n}$-endomorphism of $N$ which is non-zero, but $\psi^{2}$ is zero. Hence

$$
0 \neq \operatorname{Im}(\psi) \subseteq \operatorname{ker}(\psi) \subseteq M^{\prime}
$$

and $\psi$ induces a non-zero homomorphism from $N / M^{\prime}$ to $M^{\prime}$. But $N / M^{\prime} \cong$ $M$ has simple top $L(\lambda)$ and it follows that $L(\lambda)$ occurs in $M^{\prime}$, a contradiction. 
Next, observe a more general fact.

Lemma 3.1.5. Let $A$ be an algebra over some field, and let $0 \rightarrow M \rightarrow N \rightarrow$ $M^{\prime} \rightarrow 0$ be a short exact sequence of cyclic A-modules.

(a) Suppose there is an ideal $I$ of $A$ such that $M=I M$ and $M^{\prime}=I M^{\prime}$. Then $N=I N$.

(b) Suppose $J$ is an idempotent ideal and $J M=0, J M^{\prime}=0$. Then $J N=0$.

Proof. We may assume that $M \subset N$ and that $N \rightarrow M^{\prime}$ is the canonical map. Let $m$ be a generator of $M$ and $m^{\prime}=n+M$ a generator of $M^{\prime}$.

(a) Here, $M=I m$ and $M^{\prime}=I m^{\prime}$. Clearly, $I m+I n \subseteq N$. Conversely, let $x \in N$. Then $x+M \in M^{\prime}=I M^{\prime}=I m^{\prime}$, so there is some $z^{\prime} \in I$ such that $x+M=z^{\prime} m^{\prime}=z^{\prime} n+M$ and then $x-z^{\prime} n \in M$. Since $M=I M=I m$, there is some $z \in I$ with $x-z^{\prime} n=z m$, and hence $x \in I n+I m$. So $N=I n+I m$, and hence $N=I N$.

(b) Let $x \in N$ and $z \in J$. It is enough to show that $z x=0$. Since $J^{2}=J$, there are $u_{i}, u_{i}^{\prime} \in J$ such that $z=\sum_{i} u_{i} u_{i}^{\prime}$. We have $u_{i}^{\prime} x+M \in J M^{\prime}=0$, so $u_{i}^{\prime} x \in M$ for all $i$. Then $u_{i}\left(u_{i}^{\prime} x\right) \in J M=0$ and $z x=0$.

3.1.6. We concentrate now on the case when $M$ and $M^{\prime}$ are standard modules which are also simple. By Lemma 3.1.2 we may assume that $M \cong$ $M^{\prime}$, say $M=W(t, \alpha)$. If $N$ is an extension of $M$ by itself then by Lemma 3.1 .5 we know $N=I_{t} N$ and $I_{t-2} N=0$.

\subsection{The map $\tau_{t}$.}

Lemma 3.1.5 hints that to understand the module $N$ of 3.1.6, it helps to understand the structure of $I_{t} / I_{t-2}$ as a $D_{n}$-module. We therefore proceed by introducing a certain map, $\tau$, on $I_{t} / I_{t-2}$. The basis for $I_{t} / I_{t-2}$ which we work with consists of elements $D+I_{t-2}$ as $D$ ranges over the set of diagrams for $D_{n}$ with $t$ through-strings.

3.2.1. The invertible linear map $\tau=\tau_{t}$ on $I_{t} / I_{t-2}$ (for $t>0$ ) is defined by its effect on the basis elements $D+I_{t-2}$ via

$$
\tau\left(\left[S_{1}, S_{2}, w\right]+I_{t-2}\right)=\left[S_{1}, S_{2}, w+1\right]+I_{t-2} .
$$

3.2.2. The irreducible modules for $D_{n}$ corresponding to the section $I_{t} / I_{t-2}$ may be classified using the map $\tau_{t}$ : It follows from [6, Proposition 4.1.1] that the irreducible representation $\rho$ with label $(t, \alpha)$ satisfies

$$
\rho\left(\tau\left(\left[S_{1}, S_{2}, w\right]\right)\right)=\alpha \rho\left(\left[S_{1}, S_{2}, w\right]\right) .
$$

Lemma 3.2.3. Consider $I_{t} / I_{t-2}$ as a left (respectively, right) $D_{n}$-module in the natural way. Then $\tau$ is an automorphism of left (respectively, right) $D_{n}$-modules.

Proof. It is enough to show that $\tau$ is a homomorphism of $D_{n}$-modules. This is checked by considering properties of the diagram basis. 
3.2.4. Lemma 3.2.3 means that if $p$ is any polynomial then $p(\tau) \cdot I_{t} / I_{t-2}$ is a left and right $D_{n}$-submodule (in particular, an ideal) of $I_{t} / I_{t-2}$.

3.3. The indecomposable modules $\mathcal{I}_{M}(S)$.

We now use the map $\tau$ to construct explicit examples of self-extensions of simple standard modules.

3.3.1. Let $M$ be a simple standard module with label $(t, \alpha)$. We define the algebra $\mathcal{I}_{M}$ to be

$$
\frac{I_{t} / I_{t-2}}{(\tau-\alpha)^{2} I_{t} / I_{t-2}} .
$$

The point of introducing $\mathcal{I}_{M}$ is that the module $N$ of 3.1.6 is naturally an $\mathcal{I}_{M}$-module. The definition of $\tau$ and Lemma 3.2.3 means that it makes sense to apply $\tau$ to $I_{t} / I_{t-2}$-modules, such as $M$ and $N$ (see Lemma 3.1.5). We deduce from 3.2.2 that $(\tau-\alpha) M=0$. It therefore follows that $(\tau-\alpha)^{2} N=0$, and thus $N$ is an $\mathcal{I}_{M}$-module. Note that $\mathcal{I}_{M}$ is finite dimensional.

Lemma 3.3.2. The dimension of $\mathcal{I}_{M}$ is $2(\operatorname{dim} M)^{2}$. A basis is given by the images of the diagrams

$$
\left\{\left[S_{1}, S_{2}, r\left(S_{1}, S_{2}\right)\right]\right\} \cup\left\{\left[S_{1}, S_{2}, r\left(S_{1}, S_{2}\right)+1\right]\right\}
$$

as $S_{1}$ and $S_{2}$ range over all annular involutions in the set $I(t)$, where $M$ has label $(t, a)$ for some $a \in K^{*}$.

Proof. This follows from the observation that the image of $\left[S_{1}, S_{2}, w+2\right]$ is equal in $\mathcal{I}_{M}$ to a linear combination of the images of $\left[S_{1}, S_{2}, w+1\right]$ and $\left[S_{1}, S_{2}, w\right]$, because we are quotienting out by a quadratic in $\tau$.

Next we show that $\mathcal{I}_{M}$ decomposes as a direct sum of certain left $D_{n^{-}}$ submodules.

3.3.3. Let $S \in I(t)$. We define $\mathcal{I}_{M}(S)$ to be the subspace of $\mathcal{I}_{M}$ spanned by those basis elements (as in Lemma 3.3.2) of the form $\left[S_{1}, S, w\right]$ for some $S_{1}$ and $w$.

Lemma 3.3.4. As left $D_{n}$-modules,

$$
\mathcal{I}_{M} \cong \bigoplus_{S \in I(t)} \mathcal{I}_{M}(S)
$$

Furthermore, the direct summands are all isomorphic to each other, and each one is of dimension $2 \operatorname{dim} M$.

Proof. The first assertion holds because the analogous result for $I_{t} / I_{t-2}$ is easily seen to be true, and quotienting by $(\tau-\alpha)^{2} I_{t} / I_{t-2}$ respects this.

To prove the second assertion we claim that for any $S, S^{\prime} \in I(t)$ there exists $D \in D_{n}$ such that $\left[S_{1}, S, w\right] D=\left[S_{1}, S^{\prime}, w\right]$. This follows from the characterisation of left cells in [3, Proposition 4.5.3]. Right multiplication by the image of $D$ in $\mathcal{I}_{M}$ establishes the required isomorphism of left $D_{n^{-}}$ modules from $\mathcal{I}_{M}(S)$ to $\mathcal{I}_{M}\left(S^{\prime}\right)$. 
Lemma 3.3.5. The modules $\mathcal{I}_{M}(S)$ are indecomposable and are isomorphic to self-extensions of $M$.

Proof. We observe that $(\tau-\alpha) \mathcal{I}_{M}$ is a submodule of $\mathcal{I}_{M}$ which is isomorphic to $M$, because $(\tau-\alpha)$ and $I_{t-2}$ annihilate it, but $I_{t}$ does not, and because it has the correct dimension. A similar argument shows that the quotient module is also isomorphic to $M$.

If $\mathcal{I}_{M}(S)$ were decomposable, it would be isomorphic to $M \oplus M$, and $(\tau-\alpha)$ would annihilate it. Such is not the case. (Note that we use here the fact that $M$ is simple.)

Remark 3.3.6. There are many examples of simple standard modules. Let $K^{\prime}$ be the algebraic closure of the prime subfield of $K$, let $v \in K$ be transcendental over $K^{\prime}$ and let $0 \neq \alpha \in K^{\prime}$. Then consideration of the cellular bilinear form shows that the standard module with label $(t, \alpha)$ is simple.

\subsection{Extensions of the first type.}

We can now classify extensions between simple standard $D_{n}$-modules in the case where there are no nontrivial extensions between these modules as $D_{n}(q)$-modules. (The other cases will be examined in $\S 6$.)

Proposition 3.4.1. Let $M$ be a simple standard module with no nontrivial self-extensions as a $D_{n}(q)$-module. Let

$$
0 \rightarrow M \rightarrow N \rightarrow M \rightarrow 0
$$

be a short exact sequence where $N$ is indecomposable. Then $N$ is isomorphic to $\mathcal{I}_{M}(S)$ for some $S$, and therefore for any $S$. In particular, if $\left(u^{n}-a\right) M=$ 0 , then $\left(u^{n}-a\right) N \cong M$.

Proof. By Lemma 3.1.5 we may assume $N$ is generated as a module by some $z$. It is clear that $\left(u^{n}-a\right)^{2} N=0$. Since $N$ is indecomposable and $\left(u^{n}-a\right) N \neq 0$, Lemma 3.3.5 shows that $N$ must be equal to $\mathcal{I}_{M}(S) . z$ for some $S$. Comparison of dimensions shows that the map $x \mapsto x . z$ for $x \in \mathcal{I}_{M}(S)$ is an isomorphism of modules, so $N$ is isomorphic to $\mathcal{I}_{M}(S)$.

The observation that

$$
0 \neq\left(u^{n}-a\right) \mathcal{I}_{M}(S) \neq \mathcal{I}_{M}(S)
$$

proves the last assertion.

Theorem 3.4.2. Let $M$ and $M^{\prime}$ be simple standard modules, where $M$ is a $D_{n}(q)$-module and $M^{\prime}$ is a $D_{n}\left(q^{\prime}\right)$-module.

(a) If $\operatorname{Ext}_{D_{n}(q)}^{1}(M, M)=0$ then $\operatorname{Ext}_{D_{n}}^{1}(M, M)=K$.

(b) Suppose $M \neq M^{\prime}$. If $q \neq q^{\prime}$ or both $q=q^{\prime}$ and $\operatorname{Ext}_{D_{n}(q)}^{1}\left(M, M^{\prime}\right)=0$, then $\operatorname{Ext}_{D_{n}}^{1}\left(M, M^{\prime}\right)=0$. 
Proof. Claim (a) follows from Proposition 3.4.1.

Lemma 3.1.2 proves (b) in the case $q \neq q^{\prime}$. If $q=q^{\prime}$, Lemma 3.1.4 shows that any nontrivial extension of $M$ by $M^{\prime}$ is also an extension in the category of $D_{n}(q)$-modules. This cannot happen by hypothesis.

3.4.3. Computing $\operatorname{Ext}_{D_{n}}^{1}\left(M, M^{\prime}\right)$ for arbitrary simple modules $M$ and $M^{\prime}$ is much more difficult, but the results above help to reduce the problem for $D_{n}$ to that of the finite dimensional algebra $D_{n}(q)$. We will return to the corresponding question for $D_{n}(q)$-modules in $\S 6$.

We note that Graham and Lehrer [5] have some significant results in this direction.

\subsection{Uniserial modules.}

Proposition 3.5.1. Suppose $A$ is any algebra and $M$ is a finite dimensional A-module. Suppose that for all simple modules $S_{i}$ of $A$ which occur as composition factors of $M$ we have

$$
\operatorname{Ext}^{1}\left(S_{i}, S_{j}\right)=0 \quad(i \neq j), \quad \operatorname{Ext}^{1}\left(S_{i}, S_{i}\right) \subseteq K .
$$

Then $M$ is a direct sum of uniserial modules, and each indecomposable summand has only one type of composition factor.

Proof. Let $\bar{A}=A / \mathfrak{A}$ where $\mathfrak{A}$ is the annihilator of $M$. Then $\bar{A}$ is finite dimensional and $M$ is also an $\bar{A}$-module. By the hypothesis, we also have

$$
\operatorname{Ext}_{\bar{A}}^{1}\left(S_{i}, S_{j}\right)=0
$$

and

$$
\operatorname{Ext}_{\bar{A}}^{1}\left(S_{i}, S_{i}\right) \subseteq K
$$

By general theory (see [1]) the algebra $\bar{A}$ is Morita equivalent to a direct sum of local uniserial algebras, and then every finite dimensional indecomposable module is uniserial with only one composition factor. By the Krull-Schmidt theorem $M$ is a direct sum of indecomposables, hence the result.

Theorem 3.5.2. Let $A=D_{n}$, and let $N$ be an indecomposable A-module with a filtration by simple standard modules. Suppose $N$ belongs to the block $B_{q}$ for $q \in K^{*}$.

Then if $D_{n}(q)$ is semisimple, $N$ is uniserial with all composition factors isomorphic to $M$.

Proof. Lemma 3.1.2 shows that all composition factors of $N$ are $D_{n}(q)$ modules. Since $D_{n}(q)$ is semisimple, Theorem 3.4.2 shows that Proposition 3.5.1 applies, thus proving the assertion.

3.5.3. These uniserial modules, which may be of arbitrary length, can be constructed explicitly by the methods of $\S 3.3$. The only change is the replacement of $\left(\tau-\alpha_{t}\right)^{2}$ by $\left(\tau-\alpha_{t}\right)^{k}$ for arbitrary $k$. 
3.5.4. Similar results hold for the algebra $T L\left(\widehat{A}_{n-1}\right)$. The only difference is that $T L\left(\widehat{A}_{n-1}\right)$ has only one simple module associated to the top quotient $I_{n} / I_{n-2}$. In the case where $n$ is odd, the uniserial modules for $D_{n}$ are also uniserial modules for $T L\left(\widehat{A}_{n-1}\right)$ in the way one would expect; this is true because it holds for the standard modules (see $[6, \S 4.1]$ ). In the case where $n$ is even, the rôle of $\tau$ is played by $\tau^{2}$, but the techniques are the same.

Note that unlike the situation for ordinary Temperley-Lieb algebras, one cannot construct towers of algebras in the affine case, because $T L\left(\widehat{A}_{n-1}\right)$ is not contained in $T L\left(\widehat{A}_{n}\right)$ in any obvious way.

\section{The algebra $D_{n}^{+}$and its procellular completion.}

\subsection{The algebra $D_{n}^{+}$.}

For the purposes of $\S 4$, it is convenient to work with a subalgebra, $D_{n}^{+}$, of $D_{n}$ which is spanned by certain "positive" diagrams. These are defined as follows.

4.1.1. A diagram $[S, T, w]$, where $S, T \in \operatorname{Ann}(\mathbf{n})$ have $t>0$ fixed points each, is said to be positive if and only if $w \geq n t$. We denote the span of the images of the positive diagrams in $D_{n} / I_{0}$ together with the identity by $D_{n}^{+}$.

Proposition 4.1.2. The module $D_{n}^{+}$is a subalgebra of $D_{n} / I_{0}$.

Proof. The condition for a diagram $D$ to be positive is equivalent to the condition that each through-string contribute at least $+n$ to the winding number $w(D)$. Consider a product $D_{1} D_{2}$ in $D_{n}^{+}$. This is equal to $\delta^{x} D$ for some diagram $D$. Each through-string, $e$, in $D$ arises from the concatenation of a through-string in $D_{1}$, an (optional) intermediate section composed of horizontal edges from the bottom circle of $D_{1}$ and the top circle of $D_{2}$, and a through-string in $D_{2}$. Since $D_{1}$ (respectively, $D_{2}$ ) has at most $n / 2$ horizontal edges in the bottom (respectively, top) circle, at most $n$ edges can be involved in the optional intermediate section of $e$. Each of these contributes either $+1,-1$ or 0 to the winding number of $D$. Adding in the contributions from $D_{1}$ and $D_{2}$ shows that $e$ contributes at least $+n$ to $w(D)$, as required.

\subsection{The cellular structure of $D_{n}^{+}$.}

We now show that $D_{n}^{+}$can be given a cell datum. (This is slightly surprising since it is infinite dimensional.) To do this, we first fix $q \in K^{*}$.

4.2.1. Maintain the notation of 2.5.2. The polynomial $f_{c}^{(t, j)}(X)$ is defined for $t \in \mathcal{T}(n), 1 \leq j \leq t$ and $0 \leq c \in \mathbb{N}$ via

$$
f_{c}^{(t, j)}(X)=X^{n t}\left(X^{t}-q\right)^{c} \prod_{i>j}\left(X-r_{i}(t, q)\right) .
$$


We order the triples $(c, t, j)$ by the condition $\left(c_{1}, t_{1}, j_{1}\right)<\left(c_{2}, t_{2}, j_{2}\right)$ iff

$$
\left(-c_{1}, t_{1}, j_{1}\right)<\left(-c_{2}, t_{2}, j_{2}\right)
$$

in the obvious lexicographic order.

Proposition 4.2.2. A cell datum for $D_{n}^{+},(\Lambda, M, C, *)$, is given as follows.

(a) $\Lambda$ is the poset of triples $(c, t, j)$ given in 4.2.1.

(b) For $(c, t, j) \in \Lambda$, let $M(c, t, j)=\operatorname{Ann}(\mathbf{n}) \cap I(t)$.

(c) If $\lambda=(c, t, j)$ and $S_{1}, S_{2} \in M(\lambda)$, define

$$
C_{S_{1}, S_{2}}^{\lambda}:=f_{c}^{(t, j)}\left(S_{1}, S_{2}^{*}\right) \text {. }
$$

(d) $\left[S_{1}, S_{2}, w\right]^{*}=\left[S_{2}^{*}, S_{1}^{*}, w\right]$ where $S^{*}=w_{0} S w_{0}^{-1}$.

Proof. It is clear that $*$ is an anti-automorphism of $D_{n}^{+}$. The image of $C$ is a basis for $D_{n}^{+}$because, for a fixed $t$, there is exactly one polynomial $f_{c}^{(t, j)}$ of degree $d$ for $d \geq n t$.

There is one more axiom to check, which is the following. If $\lambda \in \Lambda$ and $S, T \in M(\lambda)$ then for all $a \in D_{n}^{+}$we have

$$
a . C_{S, T}^{\lambda} \equiv \sum_{S^{\prime} \in M(\lambda)} r_{a}\left(S^{\prime}, S\right) C_{S^{\prime}, T}^{\lambda} \bmod D_{n}^{+}(<\lambda),
$$

where $r_{a}\left(S^{\prime}, S\right)$ is independent of $T$ and $D_{n}^{+}(<\lambda)$ is the subspace of $D_{n}^{+}$ spanned by the set

$$
\left\{C_{S^{\prime \prime}, T^{\prime \prime}}^{\mu}: \mu<\lambda, S^{\prime \prime} \in M(\mu), T^{\prime \prime} \in M(\mu)\right\} .
$$

Let $\lambda=(c, t, j)$. To verify the axiom, it is enough to work in the quotient

$$
\frac{D_{n}^{+}}{I_{n}^{+}(c)}
$$

where we define $I_{n}^{+}(c):=D_{n}^{+} \cap\left\langle\left(u^{n}-q\right)^{c+1}\right\rangle$. Note that the basis arising from $C$ is compatible with this quotient, and $C_{S, T}^{\lambda}$ has nonzero image. Also note that any basis element in the ideal $I_{n}^{+}(c)$ is strictly less than $\lambda$ by definition of the order. The proof follows by the observation that the $C$-basis elements with nonzero images form a cell basis for the quotient - this is for exactly the same reason that the $q$-Jones algebras, which correspond to the case $c=0$, are cellular (see 2.5.2).

Lemma 4.2.3. For each $\lambda \in \Lambda$, there is only a finite number of $\lambda^{\prime}$ such that $\lambda \leq \lambda^{\prime}$.

Proof. This follows from the observation that the number of $\lambda=(c, t, j)$ for a fixed value of $c$ is finite. 


\subsection{The procellular algebra $\widehat{D}_{n}^{+}$.}

4.3.1. A cell datum satisfying the condition in Lemma 4.2.3, where the poset $\Lambda$ is infinite but locally finite, is said to be of profinite type, following [7, Definition 2.1.2]. It was shown in $[7, \S 2.2]$ that in this case, it makes sense to speak of an algebra whose elements are sums of basis elements $C_{S, T}^{\lambda}$ in which infinitely many basis elements may occur with nonzero coefficients, and where the multiplication carries over in the obvious way. This is known as the procellular completion, and is formally defined (following [7, §2.1]) as follows.

4.3.2. Let $\Lambda$ be the poset of a cell datum of profinite type for an algebra $A$ with base ring $R$. We denote the set of finite ideals of $\Lambda$, ordered by inclusion, by $\Pi$.

If $P \in \Pi$, we write $A_{P}$ for the cellular quotient of $A$ with basis parametrised by the set

$$
\left\{C_{P}(S, T): S, T \in M(\lambda), \lambda \in P\right\} .
$$

We write $I_{P}$ for the $R$-submodule of $A$ spanned by all elements $C(S, T)$ where $S, T \in M(\lambda)$ for $\lambda \notin P$.

The procellular completion, $\widehat{A}$, is the inverse limit of the $A_{P}$ with the obvious homomorphisms $A_{P_{1}} \rightarrow A_{P_{2}}$ whenever $P_{1} \supset P_{2}$.

Proposition 4.3.3. The procellular completion $\widehat{D}_{n}^{+}$of $D_{n}^{+}$is canonically isomorphic to the inverse limit

$$
\cdots \rightarrow \frac{D_{n}^{+}}{I_{n}^{+}(c)} \rightarrow \frac{D_{n}^{+}}{I_{n}^{+}(c-1)} \rightarrow \cdots \rightarrow \frac{D_{n}^{+}}{I_{n}^{+}(0)} .
$$

Proof. As mentioned earlier, we may identify elements of $\widehat{D}_{n}^{+}$with sums

$$
\sum r_{S, T}^{\lambda} C_{S, T}^{\lambda}
$$

where in general infinitely many of the $r_{S, T}^{\lambda}$ are nonzero. Because the $C$ basis is compatible with the ideal chain given (see the proof of Proposition 4.2.2), the isomorphism works as claimed.

\subsection{Dense subalgebras.}

4.4.1. It was shown in $[7, \S 2]$ that a base of open neighbourhoods of 0 in a procellular algebra $\widehat{A}$ is given by the ideals $\widehat{I}_{P}$, where $P$ is an ideal of $\Lambda$ and an element of $\widehat{A}$ is in $\hat{I}_{P}$ if and only if it is of the form

$$
\sum r_{S, T}^{\lambda} C_{S, T}^{\lambda}
$$

where $r_{S, T}^{\lambda} \neq 0 \Rightarrow \lambda \notin P$. 
4.4.2. With respect to this topology, $\widehat{A}$ is a complete Hausdorff topological ring with a homeomorphism $\widehat{*}$ induced by the maps $*$ on the finite dimensional cellular quotients [7, Proposition 2.2.4]. Furthermore, the original cellular algebra $A$ occurs as a dense subalgebra in the obvious way.

In the case of $D_{n}^{+}$, one can say more. The following result shows that $D_{n} / I_{0}$ is "almost cellular".

Proposition 4.4.3. The obvious map from $D_{n}$ to $\widehat{D}_{n}^{+}$identifies the quotient $D_{n} / I_{0}$ with a dense subalgebra of $\widehat{D}_{n}^{+}$.

Proof. The denseness assertion is immediate from 4.4.2 and the fact that $D_{n}^{+}$is a subalgebra of $D_{n} / I_{0}$. We need to prove the faithfulness. Consider $f \in D_{n}$ which involves only $\left[S_{1}, S_{2}, m\right], m \in \mathbb{Z}$, for fixed $S_{1}, S_{2} \in \operatorname{Ann}(\mathbf{n}) \cap I_{t}$ $(t>0)$. It suffices to show that any such $f$ maps to a non-zero element in $\widehat{D}_{n}^{+}$. Choose $k$ such that $|m|<t k$ for all $m$ such that $\left[S_{1}, S_{2}, m\right]$ occurs in $f$. Then these $\left[S_{1}, S_{2}, m\right]$ are linearly independent modulo $\left\langle\left(u^{n}-q\right)^{2 k}\right\rangle$. Observe that the elements $\left[S_{1}, S_{2}, a+b\right]$, for fixed $a$ and $0 \leq b<2 k t$, are independent in

$$
\frac{D_{n}}{\left\langle\left(u^{n}-q\right)^{2 k}\right\rangle+I_{0}},
$$

and hence in

$$
\frac{D_{n}^{+}}{I_{n}^{+}(2 k-1)}
$$

and in the inverse limit of Proposition 4.3.3. The claim now follows.

Remark 4.4.4. Notice that there are many completions of $D_{n}^{+}$, since the value of $q$ may be altered, but Proposition 4.4.3 holds in each case.

Proposition 4.4.5. Let $K_{m}$ be the ideal of $\widehat{D}_{n}^{+}$generated topologically by $I_{n}^{+}(m-1)$. Then the set $\left\{K_{m}: m \in \mathbb{N}\right\}$ is a basis of neighbourhoods of 0 in $\widehat{D}_{n}^{+}$.

Proof. Because each basis element $C_{S, T}^{\lambda}$ for $D_{n}^{+}$fails to be in the ideal $K_{m}$ for sufficiently large $m$, and the ideals $K_{m}$ are spanned (topologically) by the basis elements they contain, it follows that the set $K_{m}$ is a basis of neighbourhoods for 0 in $\widehat{D}_{n}^{+}$.

4.4.6. Recall from $[7, \S 2.3]$ that a smooth module for a procellular algebra is one whose annihilator is open. It follows from Proposition 4.4.5 that the smooth modules for $\widehat{D}_{n}^{+}$are precisely those annihilated by $I_{n}^{+}(c)$ for a sufficiently large $c$. Thus all the uniserial modules of $\S 3.5$ occur as smooth modules for $\widehat{D}_{n}^{+}$for a suitable value of $q$.

Remark 4.4.7. Finally in this section, we note that the constructions of $\S 4$ may be adapted to work for $O_{n}$ and its related algebras by making small changes to the arguments. 


\section{Standard modules for cellular algebras.}

In $\S 5$, we take $A$ to be an arbitrary finite dimensional cellular algebra. The aim is to study extensions of standard modules. Since $A$ is general, it is convenient to work without bases as much as possible, so we follow the approach in [9].

\subsection{Cell chains.}

5.1.1. The paper $[9]$ gives an equivalent definition for a cellular algebra. It first introduces the notion of a cell ideal $[\mathbf{9}, 3.2]$. The algebra $A$ is then cellular if there is a chain of ideals

$$
0=J_{0} \subset J_{1} \subset \ldots \subset J_{r}=A
$$

such that $J_{i} / J_{i-1}$ is a cell ideal in $A / J_{i-1}$. It is proved that a cell ideal $J$ satisfies one of the following. Either $J^{2}=0$, or else $J=A e A$ (and $J^{2}=J$ ) where $e$ is a primitive idempotent of $A$ fixed by the involution $*$, and moreover $e A e=K$, and multiplication in $A$ induces a bimodule isomorphism $J \cong A e \otimes_{K} e A$. In the last case, $A e$ is isomorphic to the associated standard module. Moreover, the case $J^{2}=J$ is equivalent to the condition that the associated bilinear form as in [4] is non-zero. If this is the case, we say that the associated standard module is "nice".

Remark 5.1.2. Note also that in the second case, $J$ is a heredity ideal in the sense of [2]. So if all quotients are idempotent then the algebra is quasi-hereditary.

5.1.3. In [9] the following is proved. Given any cellular algebra $B$ and any $B$-module $J$ then there is at least one cellular algebra $A$ which is an extension of $B$ by $J$, in which $J$ is a cell ideal with square zero. Hence not much can be said in general for standard modules which are not nice. However, the $q$-Jones algebras have a special property, namely that every standard module is isomorphic to a nice standard module.

\subsection{Homomorphisms between standard modules.}

5.2.1. Suppose $A$ is cellular, with cell chain $(*)$. In this section, we denote the standard module associated to $J_{i} / J_{i-1}$ by $W(i)$. The set of weights is $\Lambda=\{1,2, \cdots, r\}$, with the natural order, following [4]: Note that this is opposite to the usual order for a quasi-hereditary algebra. Suppose $J_{i} / J_{i-1}$ is idempotent. Then there is a primitive idempotent $e_{i}$ of $A$ such that $J_{i}=A e_{i} A+J_{i-1}$ and moreover $W(i) \cong A e_{i} / J_{i-1} e_{i}$. The following is a minor generalization of $[4,2.6]$, which is based on the observation that if $J$ is an idempotent ideal and $M$ is a module with $J M=0$, then for any $a \in A$ we have $\operatorname{Hom}_{A}(J a, M)=0$.

Lemma 5.2.2. Suppose $J_{i}=A e_{i} A+J_{i-1}$ where $e_{i}$ is a primitive idempotent. If $i<k$, then $\operatorname{Hom}\left(A e_{i}, W(k)\right)=0$. In particular if $W(i)$ is the associated standard module then $\operatorname{Hom}(W(i), W(k))=0$. 
Proof. The module $W(i)$ can be taken as a quotient of $A e_{i}$, and $W(k)$ is contained in $A / J_{k-1}$. Hence we have

$$
\operatorname{Hom}(W(i), W(k)) \subseteq \operatorname{Hom}\left(A e_{i}, A / J_{k-1}\right)=0,
$$

since $e_{i}$ is an idempotent, and by the observation, $e_{i}(W(k)) \subseteq e_{i}\left(A / J_{k-1}\right)=$ 0 .

5.2.3. This gives some information on repeated occurrences of standard modules. Suppose $W(i)$ is a standard module such that $J_{i}=A e_{i} A+J_{i-1}$ for a primitive idempotent $e_{i}$. If $j \neq i$ and $W(j)$ is isomorphic to $W(i)$ then by the above Lemma we have $j<i$, and moreover the associated cell quotient $J_{j} / J_{j-1}$ is nilpotent.

\subsection{Extensions of standard modules.}

Suppose $W(i)$ is a standard module such that $J_{i}=A e_{i} A+J_{i-1}$. Consider $\operatorname{Ext}^{1}(W(i), W(k))$. In case $A$ is quasi-hereditary then $\operatorname{Ext}^{1}(W(i), W(k))=0$ for $i \leq k$ (see [2]). It is natural to ask whether this generalizes to cellular algebras.

The module $W(i)$ has a projective cover

$$
0 \rightarrow J_{i-1} e_{i} \rightarrow A e_{i} \rightarrow W(i) \rightarrow 0 .
$$

Applying $(-, W(k)):=\operatorname{Hom}_{A}(-, W(k))$, this gives

$$
\begin{aligned}
0 \rightarrow(W(i), W(k)) \rightarrow\left(A e_{i}, W(k)\right) \rightarrow\left(J_{i-1} e_{i}, W(k)\right) & \\
& \rightarrow \operatorname{Ext}^{1}(W(i), W(k)) \rightarrow 0 .
\end{aligned}
$$

Suppose $i \leq k$. Then the first two terms are isomorphic, by the above Lemma if $i<k$, and by [4] for $i=k$. Hence we get for $i \leq k$ that

$$
\operatorname{Ext}^{1}(W(i), W(k)) \cong \operatorname{Hom}\left(J_{i-1} e_{i}, W(k)\right) .
$$

In general, this can be non-zero. For example, any commutative local algebra over an algebraically closed field is cellular, with $*$ the identity, and all standard modules are isomorphic to $K$ (see $[\mathbf{9}, 3.5])$. But $\operatorname{Ext}^{1}(K, K) \neq 0$ unless the algebra is 1-dimensional.

\subsection{Idempotent cell chains.}

In particular situations one can say more. The following will be used for the algebras $D_{n}(q)$.

Lemma 5.4.1. Suppose the ideals $J_{i}, J_{i-a}$ in the cell chain are idempotent. Suppose the quotient $J_{i} / J_{i-a}$ is the direct sum of copies of $M_{i}$ where $M_{i}$ is generated by a primitive idempotent of $A / J_{i-a}$ and has a filtration with quotients isomorphic to $W(i)$. Then:

(a) If $i<k$ then $\operatorname{Ext}^{1}(W(i), W(k))=0$;

(b) $\operatorname{Ext}^{1}(W(i), W(i)) \neq 0$ if and only if $M_{i}$ has at least two quotients isomorphic to $W(i)$. 
Proof. Let $U=J_{i-1} e_{i}$. By $\S 5.3$ we must look at $\operatorname{Hom}(U, W(k))$ for $i \leq k$. By the hypothesis, $M_{i}$ is the projective cover of $W(i)$ as a module over $A / J_{i-a}$, and there is an exact sequence

$$
0 \rightarrow V \rightarrow M_{i} \rightarrow W(i) \rightarrow 0,
$$

where $V$ has a filtration with quotients isomorphic to $W(i)$. The projective cover over $A$ (from 5.3) factors through this, and we get

$$
0 \rightarrow J_{i-a} e_{i} \rightarrow U \rightarrow V \rightarrow 0
$$

and an exact sequence

$$
0 \rightarrow \operatorname{Hom}(V, W(k)) \rightarrow \operatorname{Hom}(U, W(k)) \rightarrow \operatorname{Hom}\left(J_{i-a} e_{i}, W(k)\right) .
$$

Since $J_{i-a}$ is idempotent and $J_{i-a} W(k)=0$ for $i \leq k$ we have $\operatorname{Hom}\left(J_{i-a} e_{i}, W(k)\right)=0$.

(a) Assume $i<k$. Then $\operatorname{Hom}(W(i), W(k))=0$ (see Lemma 5.2.2), and since $V$ is filtered with quotients $W(i)$, it follows by induction that $\operatorname{Hom}(V, W(k))=0$ and hence that $\operatorname{Hom}(U, W(k))=\operatorname{Ext}^{1}(W(i), W(k))=0$.

(b) Let $i=k$. If $V=0$ then the extensions are zero. Otherwise, there is a surjective map from $V$ onto $W(i)$ and hence $\operatorname{Ext}^{1}(W(i), W(i))$ is nonzero.

\section{Extensions in the non-separable case.}

We will now study standard modules for the algebras $A:=D_{n}(q)$ by modifying the cell chain described in 2.5.4 and 2.5.7. The same results hold with minor modifications for the algebras $O_{n}(q), \Gamma_{n}(q)$ and also for $D_{n}^{+} / I_{n}^{+}(c)$. To keep the notation simpler we work with $D_{n}(q)$; for the modifications, see 6.3.4, 6.3.5 and 6.3.7. We are interested in the structure of the section $I_{t}(q) / I_{t-2}(q)$, where $I_{t}(q)$ is the ideal of $A$ corresponding to the ideal $I_{t}$ of $D_{n}$ which has a good description by 2.5.1.

\subsection{Direct sum decomposition of $I_{t}(q) / I_{t-2}(q)$.}

The structure of $I_{t}(q) / I_{t-2}(q)$ is governed by the polynomial $X^{t}-q$, and the filtration given by the cell basis is an analogue of the cyclic decomposition of $K[X] /\left(X^{t}-q\right)$. In order to obtain a direct sum decomposition of $I_{t}(q) / I_{t-2}(q)$ into indecomposable direct summands, we will use the primary decomposition of $K[X] /\left(X^{t}-q\right)$, whose explicit data are well-known.

6.1.1. Suppose $r_{1}, r_{2}, \ldots, r_{m}$ are the distinct roots of $X^{t}-q$. Then we have $X^{t}-q=\left(X^{m}-w\right)^{s}$ where $X^{m}-w=\prod_{i=1}^{m}\left(X-r_{i}\right)$. The ring $K[X] /\left(X^{t}-q\right)$ is the direct sum of $m$ local rings, namely

$$
\frac{K[X]}{\left(X^{t}-q\right)} \cong \bigoplus_{i=1}^{m} \frac{K[X]}{\left(X-r_{i}\right)^{s}} .
$$


The block idempotents are the cosets of

$$
q_{i}(X):=\prod_{j \neq i}\left(X-r_{j}\right)^{s} \cdot d_{i},
$$

where $d_{i}=d_{i}(X)$ is a polynomial such that $\sum q_{i}(X)=1$. (This exists since the greatest common divisor of the $\prod_{j \neq i}\left(X-r_{j}\right)^{s}(1 \leq i \leq m)$ is 1 , so the ideal they generate in $K[X]$ is the whole ring.) Moreover, for $0 \leq c \leq s-1$, let

$$
g_{i}^{(c)}(X):=\left(X-r_{i}\right)^{c} q_{i}(X) .
$$

Then the $i$-th summand has $K$-basis the cosets of $g_{i}^{(c)}(X)$ for $0 \leq c \leq s-1$. Note that we have

$$
X g_{i}^{(0)}(X) \equiv r_{i} g_{i}^{(0)}(X) \bmod g_{i}^{(1)}(X) .
$$

6.1.2. We will now give an analogous direct sum decomposition of $I_{t}(q) / I_{t-2}(q)$. For a fixed $T \in \operatorname{Ann}(\mathbf{n}) \cap I(t)$, let $U(T)$ be the space spanned by all $[S, T, k]$ where $S$ runs through the annular involutions with $t$ fixed points, and $0 \leq k \leq t-1$. This is a left module (modulo $I_{t-2}$ ) and $I_{t}(q) / I_{t-2}(q)$ is the direct sum of the $U(T)$ where $T$ runs through the annular involutions with $t$ fixed points. Moreover, all $U(T)$ are isomorphic. We now fix $T$ and study $U(T)$.

6.1.3. Suppose $S \in \operatorname{Ann}(\mathbf{n}) \cap I_{t}$ is fixed as well. Consider the space spanned by all $[S, T, k]$ in $D_{n}$. We identify this with polynomials (as in 2.4.8), via the vector space isomorphism which takes $X^{k}$ to $[S, T, k]$. This maps

$$
X^{t}-q \mapsto[S, T, t]-q[S, T, 0]=\left(u^{n}-q\right)[S, T, 0],
$$

which is zero in $A$. Hence the above map induces a vector space isomorphism of $K[X] /\left(X^{t}-q\right)$ and the span of the $[S, T, k]$ in $A$. If $f(X)$ is a polynomial, write $f(S)$ for the corresponding element $f(S, T)$.

Lemma 6.1.4. The space $U(T)$ has $K$-basis the cosets of

$$
\left\{g_{i}^{(c)}(S): S \in \operatorname{Ann}(\mathbf{n}) \cap I(t), 1 \leq i \leq m, 0 \leq c \leq s-1\right\} .
$$

Proof. First note that the number of elements equals the dimension. To show linear independence, it suffices to consider the $g_{i}^{(c)}(S)$ for a fixed $S$. Under the above identification, these correspond to the cosets of $g_{i}^{(c)}(X)$ in $K[X] /\left(X^{t}-q\right)$ which we know form a basis, hence the claim follows.

6.1.5. We have as vector spaces

$$
U(T)=\bigoplus_{i=1}^{m} Q_{i}(T),
$$

where $Q_{i}(T)$ is the span of all $g_{i}^{(c)}(S)$ for fixed $i$. 


\subsection{The modules $Q_{i}(T)$.}

It turns out that the vector space decomposition of 6.1.5 is the decomposition of $U(T)$ into indecomposable summands.

\section{Proposition 6.2.1.}

(a) The $K$-space $Q_{i}(T)$ is an A-module and it is indecomposable.

(b) There is a filtration of $Q_{i}(T)$ given by

$$
0 \subset V_{s-1} \subset \ldots \subset V_{1} \subset V_{0}=Q_{i}(T)
$$

where all the successive quotients are isomorphic. Moreover, for each $k$ we have $Q_{i}(T) / V_{s-k} \cong V_{k}$.

Proof. We first prove (a). It suffices to show that $Q_{i}(T)$ is invariant under $u$ and $E_{1}$. First, we have $u[S, T, k]=\left[S^{u}, T, k^{\prime}\right]$ where $k^{\prime}=k$ if $S$ fixes $n$, and $k^{\prime}=k+1$ otherwise, and where $S^{u}$ is the annular involution which takes $i$ to $S(i-1)+1$. In particular this shows that $k^{\prime}=k+f$ where $f=f(u, S)$ is independent of $k$. We deduce that $u \cdot g_{i}^{(c)}(S)$ is the element obtained from $X^{f} \cdot g_{i}^{(c)}(X)$ by substituting $\left[S^{u}, T, k\right]$ for $X^{k}$; this too lies in $Q_{i}(T)$.

Consider now $E_{1}[S, T, k]$, that is, $\left[S_{1}, S_{1}, 0\right] \cdot[S, T, k]$ (with $S_{1}=(12)$ ). Either this lies in $I_{t-2}$ for all $k$, or else it is equal to

$$
\delta^{\left(S_{1}, S\right)}\left[S^{\prime}, T, k+f\right],
$$

where $f=f\left(S_{1}, S\right)$ depends only on $S_{1}, S$ but not on $k$, and where $S^{\prime}$ is an element in $\operatorname{Ann}(\mathbf{n}) \cap I_{t}$. Here $\left(S_{1}, S\right)$ is the number of loops which appear in the product (see 2.1.6). (To see this, we may use the map $\tau$ from $\S 3.2$ which we know induces a module homomorphism of $I_{t}(q) / I_{t-2}(q)$. Namely, $[S, T, k]=\tau^{k}([S, T, 0])$ and $\tau$ commutes with $E_{1}$ modulo $I_{t-2}$. So the number $f$ is determined by $E_{1}[S, T, 0]$ if this is non-zero.) It follows that $E_{1} g_{i}^{(c)}(S)$ is either zero or is equal to the element obtained from $\delta^{\left(S_{1}, S\right)} \cdot X^{f} g_{i}^{(c)}(X)$ by substituting $\left[S^{\prime}, T, k\right]$ for $X^{k}$, and this again lies in $Q_{i}(T)$.

Now we will show that $Q_{i}(T)$ is indecomposable.

Let $e:=\left(\delta^{(T, T)}\right)^{-1}[T, T, 0]$. Then $e$ is an idempotent which belongs to $I_{t}$, and we have

$$
A e \equiv I_{t} e \equiv U(T) \quad \bmod I_{t-2} .
$$

It follows that the endomorphism ring of $U(T)$ is isomorphic to $e A e(\bmod$ $e I_{t-2} e$ ). This ring is spanned by the cosets of the $[T, T, k]$ and it is isomorphic to

$$
\frac{K[X]}{\left(X^{t}-q\right)}
$$

(An isomorphism is induced by $X \rightarrow\left(\delta^{(T, T)}\right)^{-1}[T, T, 1]$.) We know that this has precisely $m$ primitive idempotents and hence $U(T)$ must be the direct sum of $m$ indecomposable modules. We have already found $m$ non-zero summands, the $Q_{i}(T)$, so these must be indecomposable. 
We now tackle (b). Let $V_{d}$ be the span of $\left\{g_{i}^{(c)}(S): c \geq d, S \in \operatorname{Ann}(\mathbf{n}) \cap\right.$ $I(t)\}$. These are submodules which give a filtration of $Q_{i}(T)$. We claim that $V_{j} / V_{j+1}$ is isomorphic to $V_{0} / V_{1}$. Namely, there is a well-defined linear map which takes $g_{i}^{(j)}(S)+V_{j+1}$ to $g_{i}^{(0)}(S)+V_{1}$. The action of $u, E_{1}$ was determined above, and we see that this map is an $A$-homomorphism, hence an isomorphism. Similarly we have an $A$-homomorphism $Q_{i}(T) \rightarrow V_{k}$ which takes $g_{i}^{(c)}(S)$ to $g_{i}^{(c+k)}(S)$ and this gives an isomorphism between $Q_{i}(T) / V_{s-k}$ and $V_{k}$, as required.

Lemma 6.2.2. The quotient $Q_{i}(T) / V_{1}$ is the standard module $W(\lambda)$ with label $\lambda=\left(t, r_{i}\right)$.

Proof. Let $S_{1}, S_{2} \in \operatorname{Ann}(\mathbf{n}) \cap I(t)$ and $\omega \in Q_{i}(T) \backslash V_{1}$. Then

$$
\left[S_{1}, S_{2}, k+1\right] \omega \equiv r_{i}\left[S_{1}, S_{2}, k\right] \omega \bmod V_{1}
$$

and there are $\omega, S_{1}, S_{2}$ such that this is non-zero. This characterizes $L(\lambda)$ : See 3.2.2. (In fact, the same holds for the standard module $W(\lambda)$.)

We may assume $\omega$ is the coset of $g_{i}^{(0)}(S)$ for some $S \in \operatorname{Ann}(\mathbf{n}) \cap I(t)$. The proof then follows by the argument of (a) in the proof of Proposition 6.2.1. If $S_{1}=S_{2}=S$ then the element is non-zero.

We note that 6.2.1 and 6.2.2 prove the claim made in 2.5.6 that any standard module is isomorphic to $W(\lambda)$ for some $\lambda \in \Lambda_{0}$.

Remark 6.2.3. We remark that $Q_{i}(T)$ is the projective cover of $W(\lambda)$ as a module for $A / I_{t-2}$. Actually, $q_{i}(T)$ is a non-zero multiple of an idempotent of $A / I_{t-2}$ which is then primitive.

\subsection{Properties of standard modules for $D_{n}(q)$.}

Theorem 6.3.1. Let $\lambda=\left(t, r_{j}\right)$ and $\mu=\left(t^{\prime}, s_{i}\right)$ be weights.

(a) If $\lambda \neq \mu$ and $t \leq t^{\prime}$ then $\operatorname{Ext}^{1}(W(\lambda), W(\mu))=0$.

(b) If $X^{t}-q$ is separable then

$$
\operatorname{Ext}^{1}(W(\lambda), W(\lambda))=0 .
$$

Otherwise,

$$
\operatorname{Ext}^{1}(W(\lambda), W(\lambda))=K .
$$

Proof. Most of this follows from Lemma 5.4.1. The only part missing is the dimension of $\operatorname{Ext}^{1}(W(\lambda), W(\lambda))$ if $X^{t}-q$ has multiple roots. Here we have

$$
\operatorname{Ext}^{1}(W(\lambda), W(\lambda)) \cong \operatorname{Hom}\left(V_{1}, W(\lambda)\right),
$$

where $V_{1}$ is as in Proposition 6.2.1. The epimorphism $Q_{j}(T) \rightarrow V_{1}$ gives an inclusion

$$
\operatorname{Hom}\left(V_{1}, W(\lambda)\right) \subseteq \operatorname{Hom}\left(Q_{j}(T), W(\lambda)\right)
$$


This is 1-dimensional by Remark 6.2 .3 and since $L(\lambda)$ occurs only once in $W(\lambda)$. Hence we have

$$
\operatorname{dim}\left(\operatorname{Ext}^{1}(W(\lambda), W(\lambda))\right) \leq 1 .
$$

It is non-zero since $Q_{j}(T) / V_{2}$ is a non-split extension (the quotient of an indecomposable projective).

Lemma 6.3.2. If $A=D_{n}(q)$ then all standard modules are nice. Moreover, $A$ is quasi-hereditary if and only if $A$ contains a primitive $t$-th root of 1 for all $t \in \mathcal{T}(n)$.

Proof. By Proposition 6.2.1, all standard modules occurring in $I_{t}(q) / I_{t-2}(q)$ are isomorphic to $W(t, i)$ for $1 \leq i \leq m$ if $X^{t}-q$ has $m$ distinct roots.

The algebra $A$ is quasi-hereditary if and only if all cell quotients are idempotent, and this the case if and only if $X^{t}-q$ is separable for all $t \in \mathcal{T}(n)$.

Remark 6.3.3. We note also that $A$ cannot be semisimple unless all polynomials $X^{t}-1$ for $t \in \mathcal{T}(n)$ are separable.

6.3.4. Consider the algebra $O_{n} /\left(\omega(q) \cap O_{n}\right)$ for $O_{n} /\left(\left(\omega(q)+I_{0}\right) \cap O_{n}\right)$. Then we have exactly the same results for the standard modules, replacing the polynomial $X^{t}-q$ by either $X^{t}-q^{2}$ if $n$ is odd, or by $X^{t / 2}-q$ in case $n$ is even. (The substitution must be made as described in 2.5.1.) The statement in Theorem 6.3.1 remains true, provided in (b) the polynomial is replaced accordingly.

6.3.5. Consider the algebra $\Gamma_{n}(q)$, i.e., the affine Temperley-Lieb algebra modulo the ideal $\omega(q) \cap T L\left(\widehat{A}_{n-1}\right)$ (or $\left.\omega(q)+I_{0} \cap T L\left(\widehat{A}_{n-1}\right)\right)$. As we remarked in 2.5.9, this is also cellular, and it is clear that the ideals corresponding to $I_{t}$ (for $t<n$ ) are identical with the ideals of the algebra in 6.3.4. Moreover, $I_{n} / I_{n-2}$ is 1 -dimensional, spanned by the coset of 1 . So for $t<n$ the standard modules are the same as for the algebras in 6.3.4, and the only standard module for $t=n$ is the trivial module.

Let $A=J_{q}(n)$ and $B=\Gamma_{n}(q)$. The following is easy to see, by considering the action (see Lemma 6.2.2) and by comparing dimensions.

Lemma 6.3.6. Suppose $\lambda=(t, a)$ is a weight for $A$. Then the restriction of $W(\lambda)$ to $B$ is the standard module with label $\left(t, a^{2}\right)$ if $n$ is odd and $t<n$, or $(t, a)$ if $n$ is even and $t<n$. If $t=n$ then the restriction is $K$.

6.3.7. Similar results hold for standard modules of the algebras $D_{n}^{+} / I_{n}^{+}(c)$. Instead of $I_{t}, I_{t-2}$ we must take the ideals $I_{(a, t)}$ of $D_{n}^{+}$where $I_{(a, t)}$ is the span of

$$
\left\{C_{S_{1}, S_{2}}^{\lambda}: \lambda=(d, s, j), s \leq t, d \geq a\right\}
$$

for $a \in \mathbb{N}$ and $0 \neq t \in \mathcal{T}(n)$. The non-zero quotients induced on the factor algebra are of the form $I_{(a, t)} / I_{(a, t-2)}$ or $I_{\left(a, t_{0}\right)} / I_{\left(a+1, t_{0}\right)}$ where $t_{0}$ is the smallest member of $\mathcal{T}(n)$. One replaces the polynomial $X^{t}-q$ by $X^{n t}\left(X^{t}-\right.$ $q)^{a+1}$ or $X^{n t_{0}}\left(X^{t_{0}}-a\right)^{a+1}$. 


\subsection{Extensions of the second type.}

We have a surjective homomorphism from $\Gamma:=T L\left(\widehat{A}_{n-1}\right)$ onto $B$. This induces an inclusion

$$
\operatorname{Ext}_{B}^{1}(W(\lambda), W(\mu)) \rightarrow \operatorname{Ext}_{\Gamma}^{1}(W(\lambda), W(\mu))
$$

if $W(\lambda), W(\mu)$ are standard modules for $B$, and this subspace consists of the extensions which we called "the second type" in $\S 3$. The analogue of Theorem 6.3.1 gives the following.

Lemma 6.4.1. Let $\lambda=(t, a)$ and $\mu=\left(t^{\prime}, b\right)$. Then

$$
\operatorname{Ext}_{B}^{1}(W(\lambda), W(\mu))=0
$$

if $t \leq t^{\prime}$ and $\lambda \neq \mu$. Furthermore, $\operatorname{Ext}_{B}^{1}(W(\lambda), W(\lambda))=K$ if $t<n$ and the associated polynomial has multiple roots; otherwise, $\operatorname{Ext}_{B}^{1}(W(\lambda), W(\lambda))=0$.

This is what we can say in general, independent of the value of $\delta$.

\section{The ideal $I_{0}$ for $n$ even.}

Suppose $n$ is even, so that $u^{n}$ acts as identity on the ideal $I_{0}$. Since we want to study all finite dimensional simple modules for $D_{n}$ on which $u^{n}$ acts as identity, we must also deal with modules $M$ such that $I_{0} M \neq 0$. This was not tackled in [6], even in the case of simple modules.

\subsection{The structure of the ideal $I_{0}$.}

We start by describing a labelling for the diagrams in $I_{0}$.

7.1.1. Let $I_{n}(0)$ be the set of all permutations $S$ of $\mathbb{Z}$ which have the following properties:

(a) For all $k \in \mathbb{Z}, S(n+k)=S(k)+n$;

(b) the image of $S$ in $\mathcal{S}_{n}$ is an annular involution with no fixed points.

7.1.2. With this definition, there is a one to one correspondence between the diagrams in $I_{0}$ and triples $\left[S_{1}, S_{2}, k\right]$ where $S_{1}, S_{2} \in I_{n}(0)$ and where $k \geq 0$. Here $S_{1}, S_{2}$ describe the top and the bottom of the diagram, and $k$ is the number of infinite bands.

Remark 7.1.3. The elements $I_{n}(0)$ can also be labelled as pairs $(S, x)$ where $S \in \operatorname{Ann}(\mathbf{n}) \cap I(0)$ and $x$ is the smallest positive integer such that the seam $x+1 / 2$ does not intersect any edge of the half-diagram. Although this seems more natural, it introduces extra notation which is not relevant, so we will use the labelling in 7.1.2.

7.1.4. We start by defining ideals of $D_{n}$ contained in $I_{0}$. Let $g(X)$ be a polynomial in $K[X]$. For $S, T \in I_{n}(0)$, let $g(S, T) \in I_{0}$ be the element obtained from $g(X)$ by substituting $[S, T, k]$ for $X^{k}$. If $J$ is any ideal of $K[X]$ then we define a subspace $U_{J}$ of $I_{0}$ by

$$
U_{J}:=\operatorname{span}\{g(S, T): g(X) \in J \text { and } S, T \in \operatorname{Ann}(\mathbf{n}) \cap I(0)\} .
$$


This is an ideal of $D_{n}$. Consider the left action. We have $u \cdot g(S, T)=$ $g\left(S^{u}, T\right)$, where $S^{u}(i)=S(i-1)+1$ and we have

$$
E_{1}[S, T, k]=\delta^{\left(S_{1}, S\right)} \cdot\left[S^{\prime}, T, k+f\right],
$$

for some $S^{\prime} \in I_{n}(0)$ where $f$ does not depend on $k$. So $E_{1} . g(S, T)$ is the element obtained from

$$
\delta^{\left(S_{1}, S\right)} X^{f} \cdot g(X)
$$

by substituting $\left[S^{\prime}, T, k\right]$ for $X^{k}$.

7.1.5. If $T \in \operatorname{Ann}(\mathbf{n}) \cap I(0)$ is fixed, we have the idempotent $\left(\delta^{(T, T)}\right)^{-1}[T, T, 0]$, and we write

$$
U_{J}(T):=U_{J}[T, T, 0]=\operatorname{span}\left\{g(S, T): g \in J, S \in I_{n}(0)\right\} .
$$

Lemma 7.1.6. Suppose $M$ is a finite dimensional simple $D_{n}$-module such that $I_{0} M \neq 0$. Then there is some ideal $J$ of $K[X]$ such that $U_{J} M=0$. Moreover $u^{n}$ acts as identity on $M$.

Proof. Since $M$ is simple, we have $I_{0} M=M$. Note that $I_{0}$ is generated by all $[T, T, 0]$ for $T \in I_{n}(0)$ as a left $A$-module. (First, for any $S \in I_{n}(0)$ we have $[S, T, 0] \cdot[T, T, w]=\delta^{(S, T)} \cdot[S, T, w]$. Moreover, for any $T$ there is some $S$ such that the group $\langle S, T\rangle$ has exactly one infinite orbit on $\mathbb{Z}$, so that $[T, S, 0] \cdot[T, T, w]$ is a non-zero multiple of $[T, T, w+1]$.) So there is some $[T, T, 0]$ with $[T, T, 0] M \neq 0$. Pick and fix $m \in M$ and $T$ such that $m_{1}:=[T, T, 0] m \neq 0$. Then $M=I_{0} m_{1}$.

The elements $[T, T, k] m_{1}, k=0,1,2, \ldots$ are not all linearly independent. So there is some polynomial $f(X)$ with $f(T, T) m_{1}=0$ and it follows that $f(S, T) m_{1}=0$ for all $S \in I_{n}(0)$, and that $U_{J} m_{1}=0$ where $J$ is the ideal generated by $f(X)$. Hence $U_{J} M=U_{J} I_{0} m_{1} \subseteq U_{J} m_{1}=0$. The last part holds by Remark 2.5.10.

\subsection{Indecomposables and cell modules arising from $I_{0}$.}

7.2.1. Let $\omega(1)$ be the ideal of $D_{n}$ generated by $\left(u^{n}-1\right)$. Then $u^{n}$ acts as identity on a module $M$ if and only if $M$ is a module for the algebra $D_{n} / \omega(1)$. We have $\omega(1) \cap I_{0}=0$, so we consider $I_{0}$ as a subset (and as an ideal) of $D_{n} / \omega(1)$. This algebra is therefore infinite dimensional.

7.2.2. Fix an ideal $J$ of $K[X]$, and define an algebra

$$
D_{n}[J]:=D_{n} /\left(\omega(1)+U_{J}\right) .
$$

This is a finite dimensional algebra, and is the extension of $D_{n} /\left(\omega(1)+I_{0}\right)$ by the ideal $I_{0} / U_{J}$. By 7.2.1, every simple finite dimensional module for $D_{n}$ with $I_{0} M \neq 0$ is a module for such an algebra $D_{n}[J]$. Therefore, if we classify the simple modules for these algebras we complete the classification of the finite dimensional simple modules for $D_{n}$. We will show that the algebra is again cellular, and we will also study extensions of its standard modules. 
Lemma 7.2.3. The algebra $A:=D_{n}[J]$ is cellular.

Proof. We know that $J_{n}(1)=D_{n} /\left(\omega(1)+I_{0}\right)$ is cellular, so it suffices to show that $I_{0} / U_{J}$ has a cell chain. Let $f(X)$ be the monic generator of $J$. Since the field is algebraically closed we can factor it into linear factors, say

$$
f(X)=\prod_{i=1}^{k}\left(X-r_{i}\right)^{t_{i}},
$$

where the $r_{i}$ are taken pairwise distinct and $t_{i} \geq 1$ for all $i$. We can now see that there is a cell basis as for the $q$-Jones algebras (see 2.5.1).

In fact, we can also construct a direct sum decomposition of $I_{0} / U_{J}$ into a direct sum of indecomposable modules each of which is filtered by standard modules. This is the same as in $\S 6$.

7.2.4. As in 6.1 .1 we can write down explicit orthogonal primitive idempotents $q_{i}(X)$ of the $\operatorname{ring} K[X] /\langle f(X)\rangle, 1 \leq i \leq m$. Let $g_{i}^{(c)}(X)=(X-$ $\left.r_{i}\right)^{c} q_{i}(X)$, for $0 \leq c \leq a_{i}$. Define $Q_{i}(T)$ to be the span of all $g_{c}^{(i)}(S, T)$ for $S \in \operatorname{Ann}(\mathbf{n}) \cap I_{0}$ and $0 \leq c \leq a_{i}$. Letting

$$
U(T)=\bigoplus_{i=1}^{m} Q_{i}(T),
$$

we have a direct sum decomposition

$$
I_{0} / U_{J}=\bigoplus_{T \in I_{n}(0)} U(T)
$$

\section{Lemma 7.2.5.}

(a) The $K$-space $Q_{i}(T)$ is an A-module and it is indecomposable.

(b) There is a filtration of $Q_{i}(T)$ given by

$$
0 \subset V_{N-1} \subset \cdots \subset V_{1} \subset V_{0}=Q_{i}(T)
$$

where all the successive quotients are isomorphic. Moreover, for each $k$ we have $Q_{i}(T) / V_{N-k} \cong V_{k}$.

Proof. This is similar to the proof of Proposition 6.2.1. We omit details.

7.2.6. It follows that up to isomorphism, we obtain one standard module of $D_{n}[J]$ associated to $I_{0} / U_{J}$ for each root of $f(X)$, and hence also one simple module for each root. We label the standard module corresponding to the root $r_{i}$ by $W\left(0, r_{i}\right)$. Note that $Q_{i}(T)$ is the projective cover of $W\left(0, r_{i}\right)$.

Lemma 7.2.7. Let $\lambda$ be a weight $\left(0, r_{i}\right)$, and let $\mu=\left(t, s_{j}\right)$ for $0 \leq t$. Then we have:

(a) $\operatorname{Ext}^{1}(W(\lambda), W(\mu))=0$ for $\lambda \neq \mu$;

(b) if $r_{i}$ has multiplicity $>1$ as a root of $f$ then $\operatorname{Ext}^{1}(W(\lambda), W(\lambda))=K$, otherwise $\operatorname{Ext}^{1}(W(\lambda), W(\lambda))=0$. 
Proof. The proof is the same as that of Theorem 6.3.1 and we omit details.

Example 7.2.8. Suppose $n=4$, and consider $f=X^{N}$ for some $N \geq 1$. Then there is only one standard module up to isomorphism for the ideal $U_{J}$. It is possible for this module not to be simple, as follows. It is easy to calculate the Gram matrix of the associated bilinear form as in [5]. One finds that it has determinant $\left(\delta^{2}-2\right)^{2} \cdot \delta^{4}$. Hence we see that if $\delta^{2}=2$, the module $W(0,0)$ has a 2 -dimensional radical.

\subsection{Further properties of modules arising from $I_{0}$.}

7.3.1. If one considers $T L\left(\widehat{A}_{n-1}\right)$ instead of $D_{n}$ then the same techniques are applicable. One defines algebras $J_{1}(n)[J]$ analogous to the algebras $D_{n}[J]$. The only difference is that one has to substitute $[S, T, r(S, T)+2 k]$ for $X^{k}$ into polynomials.

7.3.2. The simple modules constructed for $I_{0}$ depend only on the root $r$ and not on the algebra. That is, if $f_{1}$ and $f_{2}$ are polynomials which have both $X-r$ as a factor the the corresponding simple (and standard) modules of the two algebras with label $(0, r)$ are isomorphic as modules for $D_{n}$ (respectively $\left.T L\left(\widehat{A}_{n-1}\right)\right)$. This can be seen by using the analogues of 6.1 .1 and Lemma 6.2.2.

7.3.3. Let $W=W(0, r)$ be a standard module as above. Then $W$ is a module for $D_{n}[J]$ (respectively, $J_{n}(1)[J]$ ) for any ideal of $K[X]$ of the form $J=(X-r)^{m}$ for $m \in\{1,2,3, \ldots\}$. In this case, Lemma 7.2 .5 provides indecomposable modules which have filtrations with all quotients isomorphic to $W$, of arbitrary length.

7.3.4. Let $n$ be even. Consider

$$
I_{0}+\operatorname{span}\{[S, T, w]: w \geq n t\} .
$$

This is a subalgebra of $D_{n}$ which contains the ideal $I_{0}$. We claim that this algebra is also cellular. Fix some $q \in K^{*}$. Then for the quotient modulo $I_{0}$, which is $D_{n}^{+}$, we take the cell basis as in $\S 4$. So it suffices to give a cell datum for $I_{0}$. Take any linear polynomial $X-r$, and let $J$ be the ideal generated by this in $K[X]$. Then we have a chain of ideals

$$
\cdots \subset U_{J^{k}} \subset U_{J^{k-1}} \subset \cdots \subset U_{J} \subset I_{0}
$$

which gives a cell basis, as before. 


\section{References}

[1] M.Auslander, I.Reiten and S.Smalø, Representation theory of Artin Algebras, Cambridge University Press, Cambridge, UK, 1994.

[2] E. Cline, B. Parshall and L.L. Scott, Finite dimensional algebras and highest weight categories, J. Reine Angew. Mathematik, 391 (1988), 85-99.

[3] C.K. Fan and R.M. Green, On the affine Temperley-Lieb algebras, Jour. L.M.S., to appear.

[4] J.J. Graham and G.I. Lehrer, Cellular algebras, Invent. Math., 123 (1996), 1-34.

[5] _ The representation theory of affine Temperley-Lieb algebras, Enseign. Math., 44 (1998), 173-218.

[6] R.M. Green, On representations of affine Temperley-Lieb algebras, in 'Algebras and Modules II', CMS Conference Proceedings, Amer. Math. Soc., Providence, RI, 24 (1998), 245-261.

[7] _ Completions of cellular algebras, Comm. Alg., to appear.

[8] V.F.R. Jones, A quotient of the affine Hecke algebra in the Brauer algebra, Enseign. Math., 40 (1994), 313-344.

[9] S. König and C. Xi, On the structure of cellular algebras, in 'Algebras and Modules II', CMS Conference Proceedings, Amer. Math. Soc., Providence, RI, 24 (1998), 365-386.

[10] P. Martin and H. Saleur, On an algebraic approach to higher dimensional statistical mechanics, Comm. Math. Phys., 158 (1993), 155-190.

[11] _ The blob algebra and the periodic Temperley-Lieb algebra, Lett. Math. Phys., 30(3) (1994), 189-206.

[12] J.C. McConnell and J.C. Robson, Non-commutative Noetherian rings, John Wiley \& Sons, 1987.

Received August 4, 1997 and revised November 13, 1998. Part of this work was done at the Newton Institute, Cambridge, and supported by the special semester on Representations of Algebraic Groups, 1997. The second author was supported in part by an E.P.S.R.C. postdoctoral research assistantship.

OXFORD UNIVERSITY

24-29 St. Giles'

OXFORD OX1 3LB

ENGLAND

E-mail address: erdmann@maths.ox.ac.uk

LANCASTER UNIVERSITY

LANCASTER LA1 4YF

ENGLAND

E-mail address: r.m.green@lancaster.ac.uk 